\title{
Postpartum Idiopathic Brachial Neuritis in a Sport Medicine Physician
}

\author{
Jessica Wade ${ }^{1}$ Taryn Taylor $^{2}$ \\ 1 University of Ottawa, Ontario, Canada \\ ${ }^{2}$ Carleton Sport Medicine Clinic, Ottawa, Ontario, Canada \\ J Brachial Plex Peripher Nerve Inj 2015;10:e50-e52.
}

\begin{abstract}
Address for correspondence Taryn Taylor, BKin, MSc, MD, CCFP, Dip Sport Med, Carleton Sport Medicine Clinic, 1125 Colonel By Drive, Ottawa, Ontario, K1S 5B6, Canada (e-mail: taryntaylor13@yahoo.com).
\end{abstract}

\author{
Abstract \\ Keywords \\ - brachial neuritis \\ - neuralgic amyotrophy \\ - Parsonage-Turner \\ syndrome
}

Idiopathic brachial neuritis is a distinctive condition that occurs most often in healthy individuals. It characteristically starts with an acute unilateral shoulder pain followed by a complex of symptoms, including weakness, paresthesias, and numbness. The cause of this syndrome is unknown and difficult to diagnose in the initial phase but may occur postsurgery, postinfection, posttrauma, or postvaccination. In this case, the patient's inciting event appeared to be a cesarean section. There was no trauma to the shoulder or upper extremity by way of positioning during the procedure. Several months after denervation of the supraspinatus and infraspinatus muscles, electromyography revealed some improvement. The diagnosis was made through a combination of clinical evaluation, ultrasound, magnetic resonance imaging, and electromyography. With the exception of weakness, this patient experienced few symptoms. Treatment is symptomatic including anti-inflammatories, opiates, and neuroleptics for pain. There is some evidence that immunotherapy may help decrease the length of symptoms, but there is no strong evidence to support steroid use. Physical therapy, the foundation of therapy in this case, is a useful adjunct for rehabilitation. In general, this condition has a good prognosis for recovery, although often slow, even when there is complete denervation. This patient made a full recovery and has no lingering symptoms.

\section{Introduction}

Postoperative shoulder pain is not an uncommon complaint in abdominal procedures. The following case report focuses on postpartum brachial neuritis in an active female. Although quite rare, it is an important diagnosis of shoulder pain and weakness that may be overlooked or misdiagnosed.

\section{Case Report}

A 35-year-old woman underwent a cesarean section with spinal anesthesia due to breech presentation. There were no complications and the patient did not sustain any shear force or traction to the brachial plexus while on the operating table. The shoulder was symptom free until 24 hours after delivery when a sharp pain developed in the right shoulder. Approxi-

received

June 20, 2014

accepted after revision

June 3, 2015

published online

July 24, 2015
$10.1055 / \mathrm{s}-0035-1558424$ ISSN $1749-7221$. mately 12 hours after the pain had begun, the patient had difficulty washing her hair owing to new-onset shoulder weakness. She was told that her symptoms were due to irritation of the diaphragm and phrenic nerve from air in the abdomen following surgery. The patient and baby were discharged after 3 days in hospital by which time the pain had subsided but the weakness persisted. She was unable to hold the car seat with her right arm and struggled opening a jar, pouring water from a jug, or throwing a ball.

When there was no improvement in strength after 6 weeks, the patient was seen by a sports medicine physician. An ultrasound was ordered to assess for blood clot and rotator cuff pathology. On physical examination, the patient had full range of motion in the neck and shoulder, and there was no pain on palpation or with resisted testing. There was

Copyright $\odot 2015$ Georg Thieme Verlag KG Stuttgart - New York
License terms

(®) $\odot \circledast$ 
Table 1 Electrodiagnositc studies performed at 7 weeks

\begin{tabular}{|l|l|l|}
\hline Type of study & Muscle examined & Results \\
\hline Median motor study & Abductor pollicis brevis & Normal \\
\hline Median sensory study & $3^{\text {rd }}$ digit & Normal \\
\hline $\begin{array}{l}\text { Lateral antebrachial } \\
\text { cutaneous sensory study }\end{array}$ & Forearm & Normal \\
\hline Needle EMG & Infraspinatus & $\begin{array}{l}\text { Denervation } \\
4+\text { positive sharp waves } \\
4+\text { fibrillation } \\
\end{array}$ \\
& & No motor units \\
\hline Needle EMG & Supraspinatus & $\begin{array}{l}3+\text { positive sharp waves } \\
3+\text { fibrillation } \\
\text { Decreased motor units } \\
\text { Few polyphasic units }\end{array}$ \\
\hline Needle EMG & & Normal \\
\hline
\end{tabular}

Abbreviation: EMG, electromyography.

significant weakness with resisted external rotation of the right shoulder and mild weakness with resisted abduction. Screening neurologic examination was normal apart from the weakness. There was no family history of neuromuscular disease. Nerve conduction studies (NCSs) and electromyography (EMG) were performed after the ultrasound was reported as normal. NCS and EMG revealed florid abnormal spontaneous activity in the supraspinatus and infraspinatus muscles ( - Table 1). Recruitment was reduced in a neurogenic pattern in the supraspinatus, with a few polyphasic motor units and no motor units were recruited in the infraspinatus.

The diagnosis of brachial neuritis was substantiated with extensive electrophysiologic testing. The affected nerve appeared to be the suprascapular nerve, given its innervation of the supraspinatus and infraspinatus muscles. Magnetic resonance imaging (MRI) showed atrophy of the affected muscles, but the brachial plexus and structures of the shoulder were reported as normal (- Fig. 1A-C).

Repeat NCS/EMG was performed 6 months postpartum. Strength testing was conducted and showed normal strength of the supraspinatus and improved strength with external rotation (infraspinatus). The NCS/EMG revealed no denervation of the infraspinatus muscle (-Table 2 ). There were many motor units present, motor unit recruitment was slightly decreased, and there were a few polyphasic units. Both clinically and electrodiagnostically, recovery was observed with improvement in strength and recruitment of motor units. The patient was told to return to normal activities and increase external rotation strength with the aid of physiotherapy.

\section{Discussion}

Idiopathic brachial neuritis, also known as neuralgic amyotrophy or Parsonage-Turner syndrome, is a rare condition that often arises in healthy individuals. ${ }^{1-4}$ Prior reports have emphasized the hereditary forms of brachial neuritis as being particularly likely to predispose to postpartum events. ${ }^{2}$ The condition typically starts with an acute event with unilateral shoulder pain followed by a complex of symptoms, including weakness, paresthesias, and numbness. ${ }^{1-5}$ The cause of this syndrome is unknown and is difficult to diagnose in its initial
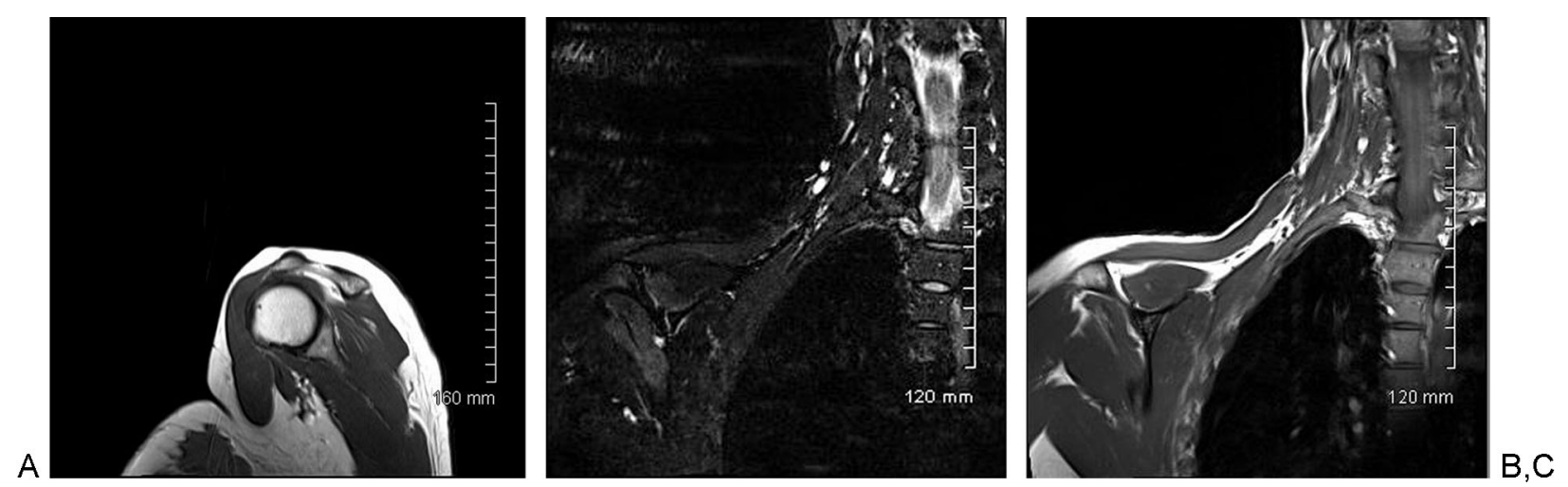

Fig. 1 (A-C) Magnetic resonance imaging of the right brachial plexus that was reported as unremarkable. There is fatty infiltration and atrophy of the infraspinatus muscle consistent with an acute on chronic denervation. Similarly, there was fatty infiltration of the supraspinatus muscle consistent with an acute denervation. 
Table 2 Electrodiagnostic studies at 6 months

\begin{tabular}{|l|l|l|}
\hline Type of study & Muscle examined & Results \\
\hline Needle EMG & Infraspinatus & $\begin{array}{l}\text { No denervation } \\
\text { Decreased motor units but several present } \\
\text { Few polyphasic units }\end{array}$ \\
\hline
\end{tabular}

Abbreviation: EMG, electromyography.

phase given its similarities to rotator cuff disease, but it often succeeds surgery, infection, trauma, or vaccination. ${ }^{1-5}$

Regarding postsurgical origins, two main theories exist. ${ }^{5}$ The first is a traction injury to the brachial plexus from improper positioning and the second is an immune-mediated inflammation of the brachial plexus. ${ }^{5}$ In this case the patient's arms were abducted to 90 degrees and were resting on arm rests. They were not strapped down, traction was not applied, and there was no positioning of the arms for an extended period of time. Once the baby was delivered, the patient was able to move her arms freely to hold the baby. Although in this case the patient symptoms were unilateral, brachial neuritis may present in both shoulders. Genetic predisposition may also play a role. ${ }^{2}$ The nerves commonly affected include suprascapular, long thoracic, anterior interosseous, axillary, and phrenic. $^{5}$ A series by Lederman and Wilbourn suggested that neither the route of delivery nor the type of anesthesia is important in causing or triggering an episode of neuralgic amyotrophy. ${ }^{2}$ Both vaginal deliveries and cesarean sections have been associated with the condition. ${ }^{2}$

Other associations with brachial neuritis are vaccinations and viruses. No vaccinations were given to the patient near the time of delivery. There was also no sign of infection. The patient had no fever, headache, muscle soreness, fatigue, or rash. The patient's condition also improved without the use of antibiotics.

EMG is performed to assess whether there is a true brachial plexopathy and, if so, to assess the degree of denervation and the denervation pattern. ${ }^{1,3}$ MRI may also be performed to rule out other potential causes such as a brachial plexus tumor and to assess for muscular atrophy. ${ }^{1,3-5}$ In this case, active and passive ranges of motion were normal making adhesive capsulitis a less likely diagnosis. Blood work was normal and D-dimer was negative to rule out blood clot. NCSs did not support a diagnosis of cervical stenosis or nerve impingement.

Prognosis is often very good even if there is complete denervation of the nerves as seen in this case. ${ }^{1-5}$ Treatment is symptomatic including nonsteroidal anti-inflammatories (NSAIDs), opiates, and neuroleptics for pain. ${ }^{1,3-5}$ Mixed results have been shown in the use of oral prednisone as a treatment of pain and weakness. ${ }^{1,3,5}$ However, there are some cases in which the administration of immunotherapy decreased the duration of symptoms. ${ }^{3,5}$ Physical therapy is also an important adjunct in the treatment of brachial neuritis to enhance range of motion and strength of the shoulder girdle, scapular stabilizers, and rotator cuff. ${ }^{1,3-5}$ Strengthening exercises of the shoulder should not commence if there is complete denervation of the muscle, but range of motion exercises are encouraged early on. Very little pain was experienced after the initial sharp shoulder pain. The mainstay of treatment in this case was physical rehabilitation. Luckily this patient made a full recovery with no significant ongoing symptoms.

Brachial neuritis is an uncommon postpartum complication. Lack of knowledge of this entity among physicians and clinical variability often result in the lack of recognition of this neurologic condition. Patients are often misdiagnosed as having musculoskeletal disorders such as a bursitis or tendinosis. Although the mechanism of peripheral nerve injury to postpartum brachial neuritis is unknown, the favorable prognosis is reassuring despite the risk of recurrence in subsequent pregnancies. Overall, this condition has an excellent outcome, despite the often severe pain and transient disability associated with the disorder.

\section{Disclosure}

The authors report no conflict of interest. Written consent was obtained from the patient described in the Case Report

\section{References}

1 Gonzalez-Alegre P, Recober A, Kelkar P. Idiopathic brachial neuritis. Iowa Orthop J 2002;22:81-85

2 Lederman RJ, Wilbourn AJ. Postpartum neuralgic amyotrophy. Neurology 1996;47(5):1213-1219

3 Miller JD, Pruitt S, McDonald TJ. Acute brachial plexus neuritis: an uncommon cause of shoulder pain. Am Fam Physician 2000;62(9): 2067-2072

4 van Alfen N. The neuralgic amyotrophy consultation. J Neurol 2007;254(6):695-704

5 Feinberg JH, Radecki J. Parsonage-Turner syndrome. HSS J 2010; 6(2):199-205 